\title{
Acercamiento a las dinámicas familiares de una comuna de Santa Marta-Colombia
}

\section{Approach to the family dynamics of a commune of Santa Marta- Colombia}

\author{
Ivone Brito-Jiménez (iD) ${ }^{1}$, María Paola Jiménez-Villamizar (iD) ${ }^{2}$, Carolina Cortina-Navarro (iD ${ }^{3}$ \\ 1. Universidad del Magdalena. Santa Marta, Colombia. Correo: ibrito@unimagdalena.edu.co - https://orcid.org/0000-0002-2474-0615 \\ 2. Universidad del Magdalena. Santa Marta, Colombia. Correo: mjimenezv@unimagdalena.edu.co - http://orcid.org/0000-0003-2264-7422 \\ 3. Universidad del Magdalena. Santa Marta, Colombia. Correo: ccortina@unimagdalena.edu.co - http://orcid.org/0000-0003-1908-952X
}

Tipología: Artículo de investigación científica y tecnológica

Para citar este artículo: Brito- Jiménez I, Jiménez-Villamizar M, Cortina- Navarro C. Acercamiento a las dinámicas familiares de una comuna de Santa martaColombia. Duazary. 2021 septiembre; 18(3 número especial): 36-43. Doi: https://doi.org/10.21676/2389783X.4261

Palabras clave: relaciones Familiares; dinámica familiar; características familiares; salud familiar.

Keywords: Family relationships;

Family dynamics; Family characteristics; Family health.

\section{RESUMEN}

El presente estudio es observacional, analítico de corte transversal, tuvo por objetivo analizar las dinámicas y funcionalidad familiar de una comuna en condición de vulnerabilidad en la ciudad de Santa Marta. Los participantes fueron 2329 sujetos entre mujeres y hombre con edades entre los 18 a 93 años, donde el $34,5 \%$ era población juventud, el $56,2 \%$ adulto y $9,3 \%$ adulto mayor, en lo que respecta a las dinámicas familiares el $12,4 \%$ se perciben estructuralmente Relacionada, frente al 10,9\% como Caóticamente Relacionada, el $51,5 \%$ de la muestra percibe que tienen una buena funcionalidad familiar, frente a $2,4 \%$ disfuncionalidad familiar severa. Se concluye que la juventud tiene una mejor percepción de las dinámicas y funcionalidad familiar.

\section{ABSTRACT}

The present study is observational, analytical, cross-sectional, and its objective was to analyze the family dynamics and functionality of a community in a vulnerable condition in the city of Santa Marta. The participants were 2329 subjects between women and men between 18 and 93 years of age, where $34.5 \%$ were youth, $56.2 \%$ adults and $9.3 \%$ older adults. Regarding family dynamics, $12.4 \%$ were perceived as structurally related, compared to $10.9 \%$ as chaotically related, $51.5 \%$ of the sample perceived that they had good family functionality, compared to $2.4 \%$ with severe family dysfunctionality. It is concluded that youth have a better perception of family dynamics and functionality. 


\section{INTRODUCCIÓN}

Las dinámicas familiares en Colombia y la Región Caribe han tenido grandes transformaciones a partir de eventos multifactoriales asociados con las relaciones inter personales de los miembros ${ }^{1}$.

La familia es la célula de la sociedad y por eso es una de las instituciones sociales claves cuando se quiere estudiar y vislumbrar los procesos de transformación social y cultural de una nación ${ }^{2}$. Todo cambio o afectación ejercida en el núcleo familiar se verá reflejado de una u otra manera en la sociedad $^{3}$.

Para realizar el análisis de las condiciones relacionales de las familias, se cuentan con diversos instrumentos de valoración que permiten el acercamiento a las realidades sociales ${ }^{4}$, en el caso de las familias entendidas como un sistema donde sus miembros se relacionan entre si formando interacciones dinámicas, donde trasmiten valores, creencias y hábitos ${ }^{5}$, además que se relacionan en un contexto social que permite conocer el nivel de funcionamiento, cohesión, adaptación y comunicación entre cada miembro permitiendo el proceso de adaptación familiar a través de su ciclo de vida, nuevas situaciones que evidencien crisis o estrés ${ }^{6}$. En este caso dichos aspectos podrán promover el funcionamiento o disfuncionalidad familiar ${ }^{7}$.

Los instrumentos de atención familiar permiten identificar en qué medida son funcionales o no y si cuentan con un recurso adecuado como soporte social ${ }^{8}$. Autores como Polaino-Lorent ${ }^{9}$, distinguen principios que facilitan el mejoramiento de las condiciones familiares en el siglo XXI tales como: la coherencia entre convicciones y comportamientos, la fidelidad entre esposos, la comunicación conyugal y familiar, el respeto por las diferencias entre intereses, valores y gustos, la conciliación entre familia y trabajo, las relaciones con la familia de origen, la educación de los hijos, la salud física y psíquica, las relaciones sociales, y la forma de administrar los recursos económicos de y para la familia.
Actualmente existen diversos mecanismos para la valoración familiar mecanismos, tales como las fichas familiares, el Ecomapa, el genograma y el APGAR familiar ${ }^{10,11}$.

En la presente investigación se analizó las dinámicas y funcionalidades familiares, entendidas las primeras como vínculos que se dan internamente en la vida doméstica a partir de relaciones de parentesco y afinidad ${ }^{12-15}$. La funcionalidad familiar basada en tres dimensiones: cohesión, adaptabilidad y comunicación, a través del modelo circunflejo de los sistemas familiar y conyugal, como elementos que componen las familias que pueden equilibrar o desequilibrarlas ${ }^{16}$.

Entendiendo la relevancia e inherencia de este tema en comunidades en condición de vulnerabilidad, se llevó a cabo la presente investigación, que tiene por objetivo analizar las dinámicas y funcionalidad familiar de una comuna en condición de vulnerabilidad en la ciudad de Santa Marta.

\section{MATERIALES Y MÉTODOS}

\section{Tipo de Investigación}

El presente estudio es observacional, analítico de corte transversal.

\section{Participantes}

La población está compuesta por personas residentes en la comuna seis - localidad dos de la ciudad de Santa Marta. Los Participantes fueron 2329 sujetos con edades comprendidas entre los 18 a 93 años.

La muestra se calculó mediante muestreo probabilístico aleatorio simple con un margen de error de $5 \%$. Se tuvo en cuenta como criterios de inclusión mayores de 18 años, residir en la comuna seis y la aceptación de participación expresa en el consentimiento informado. 


\section{Instrumentos}

Dinámicas familiares: El Faces III (Family Adaptability and Cohesion Evaluation Scales) fue validado en Colombia por Jiménez et $a l^{17}$ con un índice de confiabilidad de entre 0,82 de Alfa de Cronbach. Compuesto por 20 ítems con cinco opciones de respuesta: nunca, casi nunca, algunas veces, casi siempre, siempre, que se puntúan de 1 a 5. Las preguntas pares indican la adaptabilidad caótica puntuación de 29 a 50, flexible 25 a 28, estructurada 20 a 24, rígida 10 a 19 y las preguntas impares arrojan la cohesión, puntuaciones de 10 a 32 indican disgregada, 35 a 40 Semirelacionada, 41 a 45 relacionada y de 46 a 50 aglutinada. Para la presente investigación el Faces III presento un alfa de Cronbach de 0,79.

Funcionalidad familiar ${ }^{18}$ : para evaluar esta variable se utilizó el APGAR un cuestionario de cinco preguntas, que busca evidenciar la percepción de cada uno de los miembros a partir de la adaptabilidad, participación, gradientes de recursos, afectividad y recursos o capacidad resolutiva familiar, en un momento determinado, tiene cinco opciones: nunca, casi nunca, alguna veces, casi siempre y siempre, con puntuaciones de 0 a 4, con una puntuación total de 20 , puntuaciones entre 18 a 20 indican una buena función familiar, entre 14 y 17 disfunción familiar leve, 10 a 13 disfunción familiar moderada y de 0 a 9 puntos disfunción familiar severa. En anteriores investigaciones ${ }^{19}$ con población general se reportaron consistencias internas de 0,79. En el presente estudio el cuestionario mostro un alfa de Cronbach de 0,84.

\section{Procedimiento}

A través de las prácticas de la asignatura de Salud Familiar, se entró en contacto con las familias de la comuna seis de la ciudad de Santa Marta, se explicó el objetivo y se logró el consentimiento informado para participar en el estudio. Previo entrenamiento en el uso de los instrumentos, los cuales fueron aplicados por estudiantes de la Facultad de Ciencias de la Salud de la Universidad del Magdalena. En un intervalo de dos meses, en el periodo 2019-1, visitando casa a casa en la localidad asignada teniendo en cuenta los factores de inclusión y exclusión de participantes. Finalmente se diseñó la base de datos, se sistematizaron los cuestionarios y se procedió al análisis estadístico con el Statistical Package for the Social Sciences (SPSS versión 22).

\section{Declaración sobre aspectos éticos}

Este estudio adaptó las normas establecidas en la Declaración de Helsinki de 1975 y la Resolución 8430 de 1993 del Ministerio de Salud de Colombia para la investigación con seres humanos. Se presentó el consentimiento informado y se protegió el bienestar e integridad de los participantes, a través de la confidencialidad, derecho a la no participación retiro, devolución de resultados. La investigación fue avalada por el comité de ética de la Universidad del Magdalena REC-029-14.

\section{RESULTADOS}

En el presente estudio se contó con la participación de 2329 sujetos, con edades comprendidas entre los 18 y 93 años, de los cuales el 59,5\% eran mujeres y el $56,2 \%$ adultos (Tabla 1 ).

Tabla 1. Características sociodemográficas.

\begin{tabular}{|c|c|c|c|}
\hline \multicolumn{2}{|c|}{$\begin{array}{c}\text { Variables } \\
\text { sociodemográficas }\end{array}$} & Frecuencia & Porcentaje \\
\hline \multirow{2}{*}{ Sexo } & Hombres & 943 & $40,4 \%$ \\
\hline & Mujeres & 1386 & $59,5 \%$ \\
\hline \multirow{3}{*}{$\begin{array}{l}\text { Grupo } \\
\text { etario }\end{array}$} & $\begin{array}{l}\text { Jóvenes (18 - } \\
28 \text { años) }\end{array}$ & 804 & $34,5 \%$ \\
\hline & $\begin{array}{l}\text { Adultos (29 a } \\
59 \text { años) }\end{array}$ & 1308 & $56,2 \%$ \\
\hline & $\begin{array}{lr}\text { Adultos } & \\
\text { mayores } & \text { (60 } \\
\text { años } & \text { en } \\
\text { adelante) } & \end{array}$ & 217 & $9,3 \%$ \\
\hline
\end{tabular}

En lo referente a la cohesión y adaptabilidad el $12,4 \%$ perciben a sus familias estructuralmente Relacionada, frente al $10,9 \%$ que se encuentra Caóticamente Relacionada; permitiendo analizar las dificultades familiares en su interacción y la de sus miembros. De acuerdo a la aplicabilidad del Apgar, que analiza la funcionalidad familiar el $51,5 \%$ indican que tienen una buena funcionalidad familiar, frente a al $2,4 \%$ que identifican una disfuncionalidad familiar severa (Tabla 2). 
Tabla 2. Dinámicas y funcionalidad familiar.

\begin{tabular}{|c|c|c|c|}
\hline & & Frecuencia & Porcentaje \\
\hline \multirow{16}{*}{$\begin{array}{l}\text { Dinámica Familiar Fases III - Cohesión } \\
\text { y adaptabilidad }\end{array}$} & Caóticamente Aglutinada & 212 & $9,1 \%$ \\
\hline & Caóticamente Disgregada & 76 & $3,3 \%$ \\
\hline & Caóticamente Relacionada & 253 & $10,9 \%$ \\
\hline & Caóticamente Semirelacionada & 162 & $7,0 \%$ \\
\hline & Estructuralmente Aglutinada & 128 & $5,5 \%$ \\
\hline & Estructuralmente Disgregada & 83 & $3,6 \%$ \\
\hline & Estructuralmente Relacionada & 288 & $12,4 \%$ \\
\hline & Estructuralmente Semirelacionada & 198 & $8,5 \%$ \\
\hline & Flexiblemente Aglutinada & 148 & $6,4 \%$ \\
\hline & Flexiblemente Disgregada & 77 & $3,3 \%$ \\
\hline & Flexiblemente Relacionada & 282 & $12,1 \%$ \\
\hline & Flexiblemente Semirelacionada & 180 & $7,7 \%$ \\
\hline & Rígidamente Aglutinada & 41 & $1,8 \%$ \\
\hline & Rígidamente Disgregada & 42 & $1,8 \%$ \\
\hline & Rígidamente Relacionada & 86 & $3,7 \%$ \\
\hline & Rígidamente Semirelacionada & 73 & $3,1 \%$ \\
\hline \multirow{4}{*}{ APGAR - Funcionalidad Familiar } & Buena funcionalidad familiar & 1199 & $51,5 \%$ \\
\hline & Disfuncionalidad familiar leve & 775 & $33,3 \%$ \\
\hline & Disfuncionalidad familiar moderada & 299 & $12,8 \%$ \\
\hline & Disfuncionalidad familiar severa & 56 & $2,4 \%$ \\
\hline
\end{tabular}

Tabla 3. Dinámicas familiares según grupo etario y género.

\begin{tabular}{|l|l|l|l|l|l|}
\hline \multirow{2}{*}{ Diagnóstico del Faces III } & Grupos Etarios & \multicolumn{2}{l|}{ Sexo } \\
\cline { 2 - 6 } & Juventud & Adulto & Adulto Mayor & Hombres & Mujeres \\
\hline Caóticamente Aglutinada & 56 & 125 & 31 & 79 & 133 \\
\hline Caóticamente Disgregada & 29 & 41 & 6 & 33 & 43 \\
\hline Caóticamente Relacionada & 78 & 154 & 21 & 101 & 152 \\
\hline Caóticamente Semirelacionada & 62 & 81 & 19 & 68 & 94 \\
\hline Estructuralmente Aglutinada & 33 & 86 & 9 & 10 & 73 \\
\hline Estructuralmente Disgregada & 42 & 31 & 10 & 30 & 53 \\
\hline Estructuralmente Relacionada & 110 & 159 & 19 & 120 & 168 \\
\hline Estructuralmente Semirelacionada & 79 & 101 & 18 & 69 & 129 \\
\hline Flexiblemente Aglutinada & 38 & 93 & 17 & 31 & 98 \\
\hline Flexiblemente Disgregada & 29 & 43 & 5 & 121 & 46 \\
\hline Flexiblemente Relacionada & 100 & 154 & 28 & 80 & 161 \\
\hline Flexiblemente Semirelacionada & 74 & 88 & 18 & 15 & 100 \\
\hline Rígidamente Aglutinada & 10 & 29 & 2 & 19 & 26 \\
\hline Rígidamente Disgregada & 22 & 17 & 3 & 38 & 23 \\
\hline Rígidamente Relacionada & 22 & 60 & 4 & 34 & 48 \\
\hline Rígidamente Semirelacionada & 20 & 46 & 7 & 39 \\
\hline
\end{tabular}

Tabla 4. Funcionalidad familiar según grupo etario y género.

\begin{tabular}{|l|l|l|l|l|l|}
\hline \multirow{2}{*}{ Apgar Puntuación Total } & Grupos Etarios & \multicolumn{2}{l|}{ Sexo } \\
\cline { 2 - 7 } & Juventud & Adulto & Adulto Mayor & Hombre & Mujeres \\
\hline Buena funcionalidad familiar & 364 & 717 & 118 & 492 & 707 \\
\hline Disfuncionalidad familiar leve & 283 & 431 & 61 & 322 & 453 \\
\hline Disfuncionalidad familiar moderada & 131 & 132 & 36 & 101 & 198 \\
\hline Disfuncionalidad familiar severa & 26 & 28 & 2 & 28 & 28 \\
\hline
\end{tabular}


En lo que respecta a las dinámicas familiares (Fases III) se observa que tanto jóvenes como adultos perciben a sus familias como estructuralmente relacionadas, mientras que los adultos mayores como caóticamente aglutinada. En lo que refiere al sexo las mujeres identifican a sus familias como flexiblemente relacionadas y los hombres como estructuralmente relacionadas.

Referente a la funcionalidad familiar observamos que no hay diferencia entre los jóvenes, adultos y adulto mayor, ni en mujeres y hombres todos perciben una buena funcionalidad familiar (Tabla 4).

\section{DISCUSIÓN}

En la presente investigación se evidenció que el $12,4 \%$ de los participantes identifican a sus familias como estructuralmente relacionadas y el $3 \%$ rígidamente semirrelacionada. Los datos anteriores se pueden contrastar con investigaciones realizadas en diferentes países de Suramérica. Sigüenza et a ${ }^{20}$ trabajaron con 153 padres de familias ecuatorianos y encontraron en lo que respecta a la adaptabilidad que el $29,4 \%$ las perciben como caóticas y en cohesión el 39,5\% como unidas y el tipo de familia predominante fue caóticamente enredada con un 31,4\%. En otra investigación con 195 estudiantes de colegio se halló que el $21,5 \%$ reconocen a sus familias como estructuralmente relacionada, el $3,5 \%$ flexiblemente aglutinada ${ }^{21}$.

El 39\% de las familias evaluadas tienen una cohesión familiar relacionada caracterizada por una cercanía emocional, compromiso y distancia permitida, límites claros que reconocen la cercanía entre padres e hijos ${ }^{22}$. El 30\% una adaptabilidad caótica lo que refiere a que hay una falta de responsabilidad y de limites familiares. Donde no hay normas y las reglas cambian constantemente ${ }^{23}$. Datos muy parecidos a los hallados por Pérez ${ }^{24}$ donde el $39,5 \%$ tienen una cohesión relacionada y en la adaptabilidad un $43,1 \%$ se identifican como estructuradas. En otra investigación con estudiantes de medicina colombianos se encontró mayor puntuación en cohesión semirrelacionada (38\%) y la más baja en $16 \%$ aglutinada, correspondiente a los resultados de adaptabilidad familiar, obtenida de los datos de la encuesta, casi la mitad de los encuestados (47\%) presentó un tipo de adaptabilidad caótica, y en menos cantidad (7\%) una adaptabilidad rígida ${ }^{25}$.

De acuerdo a la aplicabilidad del Apgar, que analiza la funcionalidad familiar el $51,5 \%$ perciben que tienen una buena funcionalidad familiar, frente a al $2,4 \%$ con una disfuncionalidad familiar severa, datos que difieren a los encontrados en un estudio realizado en Barranquilla ${ }^{26}$, con 178 familias de escasos recursos en situación de vulnerabilidad, donde el $54,4 \%$ de ellas tenía algún grado de disfuncionalidad discriminada. En familias en condición de vulnerabilidad en Tolima se hallaron datos parecido a los del presente estudio donde los participantes indicaron buena función familiar en un $44,4 \%$ representado a través de la satisfacción con su entorno familiar mediante el afecto, recursos, adaptación, ganancia y participación ${ }^{27}$.

Resultados referentes a la cohesión y adaptabilidad familiar (Fases III) de acuerdo a los grupos etarios identificados en la comuna seis de la ciudad de Santa Marta, se encontró que los jóvenes y adultos reconocen a sus familias como estructuralmente relacionadas, mientras que los adultos mayores como caóticamente aglutinados, datos que difieren en una investigación con adolescentes escolarizados donde el $50 \%$ de las familias se ubican en el nivel de adaptabilidad rígido o caótico, indicando así la imperiosa influencia que ejerce el núcleo familiar sobre el adolescente, en el presente estudio se ve mayor impacto en la población adulta ${ }^{28}$. En lo que refiere al sexo las mujeres identifican a sus familias como flexiblemente relacionadas y los hombres como estructuralmente relacionadas, datos que difieren con lo hallado en estudiantes mexicano donde los hombres identificaron sus familias mayormente flexibles y con buena comunicación en sus hogares ${ }^{29}$.

Referente a la funcionalidad familiar se observó que no hay diferencia entre los grupos etarios y por el sexo todas perciben una buena funcionalidad familiar. Totalmente opuesto en jóvenes adultos quienes tenían familias con algún grado de disfunción, 2/5 partes provenían de fuera de Bogotá y $1 / 5$ parte viven solos encuentran algún grado de 
disfunción familiar sobre todo por las condiciones de las familias y sus entornos ${ }^{30}$.

La dinámica familiar goza de un importante interés $y$ es objeto de permanente estudio en las agendas de los países americanos de cara a las funciones esenciales de la salud pública que fueron revisadas por la Organización Panamericana de la Salud ${ }^{31}$. Es una necesidad realizar análisis de los roles que tienen cada una de las personas en su núcleo familiar, esto facilita a partir de los resultados obtenidos, la necesidad de plantear acciones y estrategias de carácter individual y colectivo y específicas desde un enfoque diferencial y poblacional; ajustándonos a las políticas públicas vigentes.

\section{AGRADECIMIENTOS}

Agradecimientos a la comunidad y los diferentes actores sociales que permitieron la aplicación de los instrumentos de valoración familiar, el acceso a sus viviendas y permitirnos realizar análisis de la influencia de la familia como eje protector en el desarrollo de las personas.

\section{DECLARACIÓN SOBRE CONFLICTOS DE INTERESES}

Los autores declaran que no existe conflicto de intereses.

\section{CONTRIBUCIÓN DE LOS AUTORES}

Primer autor y tercer autor: trabajo de campo y redacción.

Primer y segundo autor: diseño del estudio, análisis estadístico, escritura, revisión final y aprobación.

\section{REFERENCIAS BIBLIOGRÁFICAS}

1. Wallerstein N. Powerless, Empowerment, and Health: Implications for Health Promotion Programs. Am J. of Health Promotion. 1992; 6(3):197-205. Doi: https://Doi.org/10.4278/08901171-6.3.197.

2. Berenguer $M C$, Pérez $A$, Dávila $M$, Sánchez JI. Determinantes sociales en la salud de la familia cubana. MEDISAN. 2017; 21(1):61-73. Disponible en:

http://www.medisan.sld.cu/index.php/san/article/ view/680

3. Instituto Colombiano de Bienestar Familiar. La familia: el entorno protector de nuestros niños, niñas y adolescentes colombianos. Colombia; 2013. Disponible en: https://www.icbf.gov.co/sites/default/files/publica cion-47-a.pdf

4. Vergara C, Torres M. Familia como modelo viable de sistemas /la eficiencia de la organización más allá de la estructura: teoría para un modelo de Salud Familiar en tensión. Braz. J. of Develop. 2019; 5(9): 16498-16513.

Doi: https://Doi.org/10.34117/bjdv5n9-197

5. Díaz S, Tirado L, Simancas M. Validez de constructo y confiabilidad de la APGAR familiar en pacientes odontológicos adultos de Cartagena, Colombia. Rev. Univ. Ind. Santander Salud. 2017; 49(4):

541-548.

Doi: https://Doi.org/10.18273/revsal.v49n4-2017003

6. Herrera Santí PM. La familia funcional y disfuncional, un indicador de salud. Rev Cubana Med Gen Integr. 1997; 13(6): 591-595. Disponible en:

http://scielo.sld.cu/scielo.php?script=sci_arttext\&p id=S0864-21251997000600013\&lng=es.

7. López MA. Percepción de la funcionalidad familiar en mujeres con alteraciones psicológicas post mastectomía secundaria a cáncer de mama de la UMF 1 del IMSS delegación Agua Calientes [tesis de especialización]. Perú; Universidad Autónoma de Aguas Calientes; 2018. Disponible en: http://bdigital.dgse.uaa.mx:8080/xmlui/bitstream/ handle/11317/1684/436335.pdf?sequence=1\&isAll owed $=y$

8. Arias CL. El APGAR familiar en el cuidado primario de salud. Colombia Médica. 2014; 25(1): 26-28. Doi: https://Doi.org/10.2510/colomb.med.v25i1.1776

9. Polaino-Lorent A. Diez principios relevantes para la mejora de la familia en el siglo XXI. Revista Berit 
Internacional. 2016. 6: 53-57. Disponible en: https://1library.co/document/y4w87j0q-diezprincipios-relevantes-mejora-familia-aquilinopolaino-lorente.html

10. Ávila G. Los instrumentos y técnicas como cuestiones indisolubles en el corpus teóricometodológico del accionar del Trabajador Social. Rev Margen. 2017. 86: 1 -10. Disponible en: https://www.margen.org/suscri/margen86/avila_8 6.pdf

11. Castro-Moreno S, Freyle-Salazar YV, FuentesJimenez MF. Instrumentos de salud familiar: herramienta para detectar relaciones en familia sobre tendencias a adoptar conductas de riesgo para la salud en la comunidad Barrio Abajo, Norte Centro Histórico, Barranquilla- 2018-1 [Pregrado]. Barranquilla: Universidad Simón Bolívar; 2018. Disponible en: http://hdl.handle.net/20.500.12442/2232

12. Smilkstein G. The family APGAR: Aproposal for a family function test and its used by physicians. J Fam Pract 1978; 6(6):1231-9. Disponible en: https://pubmed.ncbi.nlm.nih.gov/660126/

13. Alviar M, Alzate A, Bedoya L, Bravo L, Cano C, Gallón $A$, et al. Caracterización, dinámica interna y procesos llevados con algunas familias de los menores infractores o en situación de peligro de los programas Despertar-es y PROAM [Tesis de especialización]. Medellín: Universidad Pontificia Bolivariana. 2006. Disponible en: https://revistascientificas.cuc.edu.co/culturaeduca cionysociedad/article/download/1049/pdf_223/

14. García B, Oliveira O. Mujeres jefas de hogar y su dinámica familiar. Pap. Poblac. 2005; 11(43): 29-51. Disponible en: http://www.scielo.org.mx/scielo.php?script=sci_art text\&pid=S1405-74252005000100002

15. Torres L, Ortega P, Garrido A, Reyes A. Dinámica familiar en familias con hijos e hijas. Revista Intercontinental de Psicología y Educación. 2007; 10(2):31-56.

Doi: https://Doi.org/10.1080/02103702.2014.996404
16. Olson DH. Curvilinearity Survives: The World Is Not Flat. Family Process. 1994; 33: 471-478. Doi: https://Doi.org/10.1111/j.1545-5300.1994.00471.x

17. Jiménez $L$, Lorence $B$, Hidalgo $B V$, Menéndez $S$. Análisis factorial de las escalas FACES (Family Adaptability and Cohesion Evaluation Scales) con familias en situación de riesgo psicosocial. Univ Psychol. 2017; 16(2): 1-12. Doi: https://Doi.org/10.11144/Javeriana.upsy16-2.afef

18. Arias L, Herrera JA. El APGAR familiar en el cuidado primario de la salud. Colombiana Med. 1994; 25:26-8. Disponible en: https://colombiamedica.univalle.edu.co/index.php /comedica/article/view/1776

19. Bellón-Saameño JA, Delgado-Sánchez A, Luna J, Lardelli-Claret $P$. Validez y fiabilidad del cuestionario de función familiar APGAR-familiar. Aten Primaria. 1996; 14(18): 289-296. Disponible en: https://www.elsevier.es/es-revista-atencionprimaria-27-articulo-validez-fiabilidad-cuestionariofuncion-familiar-apgar-familiar-14357

20. Sigüenza $W$, Buñay $R$, Guamán-Arias $M$. Funcionamiento familiar real e ideal según el modelo Circumplejo de Olson. Maskana. 2017;8: 77$85 . \quad$ Disponible en: https://publicaciones.ucuenca.edu.ec/ojs/index.ph $\mathrm{p} /$ maskana/article/view/1878

21. Aguilar C. Funcionamiento familiar según el modelo circumplejo de Olson en adolescentes tardíos [Pregrado]. Universidad de Cuenca; 2017. Disponible en: https://dspace.ucuenca.edu.ec/bitstream/1234567 89/28397/1/Trabajo\%20de\%20Titulaci\%C3\%B3n.p df

22. Sigüenza W. Funcionamiento familiar según el modelo circumplejo de Olson [Maestría]. Universidad de Cuenca; 2015. Disponible en: https://dspace.ucuenca.edu.ec/bitstream/1234567 89/21878/1/TESIS.pdf

23. Bergerman S. Cohesión y adaptabilidad familiar en estudiantes del VII ciclo de un colegio parroquial y uno estatal, Chiclayo 2016. Universidad Católica 
Santo Toribio de Mogrovejo, 2017. Disponible en: http://hdl.handle.net/20.500.12423/1223

24. Pérez L. Funcionamiento familiar prevalente en estudiantes de nivel secundaria de instituciones educativas, nuevo Chimbote, 2017 [Pregrado]. Universidad Católica de los Ángeles Chimbote; 2018. Disponible en: http://repositorio.uladech.edu.pe/bitstream/handl e/123456789/3225/FUNCIONAMIENTO\%20FAMILI AR_INSTITUCION\%20EDUCATIVA_PEREZ_\%20CHAU CA_LEYLA_LUCIA.pdf?sequence $=1 \&$ isAllowed $=y$

25. Acosta $P$, Bohórquez L. Relación entre funcionamiento familiar y desempeño académico en estudiantes de IV semestre de medicina de la universidad de ciencias aplicadas y ambientales [Pregrado]. Universidad De Ciencias Aplicadas Y Ambientales. 2016. Disponible en: https://repository.udca.edu.co/bitstream/11158/4 74/1/Trabajo\%20de\%20Grado\%20Final\%20Funcion alidad\%20familiar\%20-\%20Promedio.pdf

26. Navarro LE, Barceló MR, Rosales AM, Mejía MJ, Caro de PS, Yépez CF. Factores asociados a la funcionalidad familiar en el barrio Ciudad Modesto. Barranquilla. 1996. Disponible en: http: www.uninorte.edu.co/apoyo/Proyectos./Proyectos -UNI./Investigaciónll

27. Portillo J, Reyes-Godoy, J. Tipificación del tipo de disfunción familiar de un barrio vulnerable de la ciudad de Ibagué-Tolima, Revista Poiésis. 2014; 27, 1-11. Disponible en: http://www.funlam.edu.co/revistas/index.php/poi esis/index

28. Moreno-Olarte $\mathrm{E}$, Rodríguez-Pérez $\mathrm{K}$, VargasMancera Kelly. Relación entre los factores de cohesión, adaptabilidad familiar y género con el grado de depresión que presentan los estudiantes de 13 a 15 años de edad de Instituciones educativas privadas de la ciudad de Santa Marta [Trabajo de pregrado]. Santa Marta; Universidad del Magdalena: $2008 . \quad$ Disponible en: http://repositorio.unimagdalena.edu.co/jspui/hand le/123456789/635
29. Gallegos-Guajardo Julia, Ruvalcaba-Romero Norma A., Castillo-López Jesús, Ayala-Díaz Paulina C. Funcionamiento familiar y su relación con la exposición a la violencia en adolescentes mexicanos. Acción psicol. 2016; 13(2): 69-78. Disponible en: http://scielo.isciii.es/scielo.php?script=sci_arttext\& pid=S1578-908X2016000200069\&Ing=es. http://dx.Doi.org/10.5944/ap.13.2.17810.

30. Mora S, Moreno C. Autopercepción de salud y de red de apoyo y funcionalidad familiar, de los estudiantes que inician posgrados de medicina. Repert. Med. Cir. 2016; 25(1): 8-14. Doi: https://Doi.org/10.1016/j.reper.2016.02.005

31. Organización Panamericana de la Salud. Resolución CD42.R14. Funciones Esenciales de Salud Pública. $42 \circ$ Consejo Directivo de OPS. Washington DC, 25-29 de Septiembre, 2000. Disponible en: https://iris.paho.org/bitstream/handle/10665.2/14 23/CD42. R14sp.pdf?sequence $=2$ \&isAllowed $=y$ 